\title{
Time-resolved techniques as probes for the laser ablation process
}

\author{
Marc Hauer $^{\mathrm{a}}$, D.J. Funk ${ }^{\mathrm{b}}$, T. Lippert ${ }^{\mathrm{a}, *}$, A. Wokaun ${ }^{\mathrm{a}}$ \\ ${ }^{a}$ General Energy Research, Paul Scherrer Institut, 5232 Villigen PSI, Switzerland \\ ${ }^{\mathrm{b}}$ DX-2, MS C920, Los Alamos National Laboratory, Los Alamos, NM 87545, USA
}

Received 1 December 2003; received in revised form 1 March 2004; accepted 12 March 2004

Available online 29 July 2004

\begin{abstract}
Laser material processing is an increasing market although many processes are still not yet fully understood. It was repeatedly emphasized that a better understanding of the transient processes can help to improve the understanding of the ablation mechanism. Examples using ns-surface interferometry, ns-shadowgraphy and emission spectroscopy are shown to illustrate how these techniques can be used to obtain new insights in the laser ablation processes.

Time-resolved surface interferometry probes the remaining polymer, while ns-shadowgraphy and plasma spectroscopy observe the ablation products. Ns-shadowgraphy can be used for fluences just above the ablation threshold, to the fluences where a plasma is observed, which is probed by emission spectroscopy.

Time-resolved ns-surface interferometry of a specially designed triazene polymer reveals that the surface morphology changes and thereby the surface removal occurs only during the laser pulse. Ns-shadowgraphy is used to observe the shockwave and the plume of fragments, which are generated during laser ablation. A comparison of the ablation properties of an energetic polymer at two different wavelengths $(1064$ and $193 \mathrm{~nm})$ shows that the ejection of non-gaseous fragments (solid or liquid) is only detected after irradiation with $1064 \mathrm{~nm}$. At higher laser fluences, plasma is formed, and the atomic $(\mathrm{H})$, and diatomic species $(\mathrm{CN}, \mathrm{CH}$ and $\mathrm{C}_{2}$ ) are identified.

(C) 2004 Elsevier Ltd. All rights reserved.
\end{abstract}

Keywords: Laser ablation; Polymers; Time resolved measurements

*Corresponding author. Tel.: +41-56-310-4076; fax: +41-56-310-2199.

E-mail address: thomas.lippert@psi.ch (T. Lippert). 


\section{Introduction}

Material processing with lasers is being used since several decades, but the mechanism remains still unclear for many processes. The process of material removal of polymers when an intense laser beam irradiates the surface has been studied now for over 20 years and remains still controversial. This process, called laser ablation, is interesting for a wide range of applications (i.e. laser plasma thruster [1,2] or via holes in multi-chip modules [3]).

It was repeatedly emphasized that a better understanding of the transient processes can help to improve the understanding of the mechanism. Various time-resolved techniques (i.e. time-resolved Raman spectroscopy [4,5], time-resolved infrared spectroscopy [6,7], time-resolved quadropole mass spectroscopy [8-11]) have been applied, resulting in new information about the chemical and physical processes involved in ablation. In this paper, additional time resolved methods, i.e. ns-surface interferometry, ns-shadowgraphy, and emission spectroscopy are presented and it will be shown how these data can be used for a better understanding of the ablation process of polymers.

Ns-surface interferometry allows time-resolved thickness measurements during and after irradiation with a laser pulse, and gives thereby valuable information about the surface displacement during the ablation process. This method focuses on the structuring of the remaining polymer and is therefore used mostly in the lower fluence range (at fluences above $1 \mathrm{~J} \mathrm{~cm}^{-2}$, the ablation rates are in most cases, quite high, making the data evaluation very difficult). Previous studies using this technique have shown that some polymers exhibit a pronounced swelling and a delayed material removal [12,13], whereas other polymers revealed structuring without swelling [14-17].

Ns-shadowgraphy can be used to image the material, which is ejected after laser irradiation $[13,18,19]$. This method can be used from fluences close to the threshold up to the fluence which creates plasma. When laser ablation occurs in an atmosphere, the instantaneous released energy will form a shockwave, which is visible in the shadowgraphy images. The propagation speed of the shockwave may be applied as an indicator for the amount of gaseous products [15] and for the energy [20], which is released during the ablation process.

Time-resolved emission spectroscopy can give information about the composition [2123] and the temperatures inside the plasma, which is formed at high irradiation fluences.

The ablation process of two different polymers was studied in detail by combining data from all of these techniques. The following polymers were studied: a special designed triazene polymer, which exhibits excellent laser ablation characteristics (sharp ablation edges, no debris, low threshold fluence and high etch rates at low fluences $[24,25]$ ) and an energetic polymer, which performed very well in laser plasma thruster experiments [26].

\section{Experimental}

The ns-surface interferometry samples were prepared by spin coating the triazene from a $10 \%$ chlorobenzene solution. Solvent casting was used for the preparation of 
the films for ns-shadowgraphy and emission spectroscopy where the surface quality is less critical. A solution of the energetic polymer was mixed with carbon (to induce absorption in the IR) and a crosslinker. The solution was solvent cast and crosslinked at elevated temperatures.

The pump-probe set up for ns-surface interferometry is shown in Fig. 1. An ArF (193 nm, Lambda Physik LPX 301i, FWHM $25 \mathrm{ns)}$ or a XeCl (308 nm, Lambda Physik Compex 205, FWHM $30 \mathrm{~ns}$ ) excimer laser are used as pump lasers. The laser light of the pump laser passes through a pinhole and is imaged onto the substrate. The second harmonic $(532 \mathrm{~nm})$ of a Nd:YAG (Quantel, Brillant B, FWHM $5 \mathrm{~ns}$ ) laser was applied as a probe laser. The time resolution is achieved by using a delay generator (SRS, DG 500) between the pump and the probe laser. The probe beam is used in a Michelson interferometer setup, where the beam is divided (50/50) by a beamsplitter. One beam is reflected by the surface of a wedged glass substrate, while the other beam is reflected from the polymer surface. Both beams are recombined in the same beamsplitter and create there an interference pattern. When the film thickness of the polymer changes, the length of the optical path changes and a fringe shift results. Wedged substrates are used to allow the selection of the interface from which the probe beam is reflected. The reflection from the glass/polymer interface is neglected due to the small difference between the refractive index of the glass and the polymer. Therefore, only the reflection from the polymer/atmosphere interface can be utilized. Two variations of this setup were tested and compared:

(a) Pump and probe from the same side: The probe beam is reflected directly from the air/polymer interface (Fig. 1(a)) and the pump beam irradiates the sample from the same side, in an angle of about $60^{\circ}$. This has the disadvantage that the probe beam passes through the ablation plume and is thereby influenced by the released shockwave and the non-gaseous fragments. It is very difficult to take the effect of the shockwave and the absorption of probe beam into account. The advantage of this method is that the observed phase shift is not influenced by the irradiated polymer.

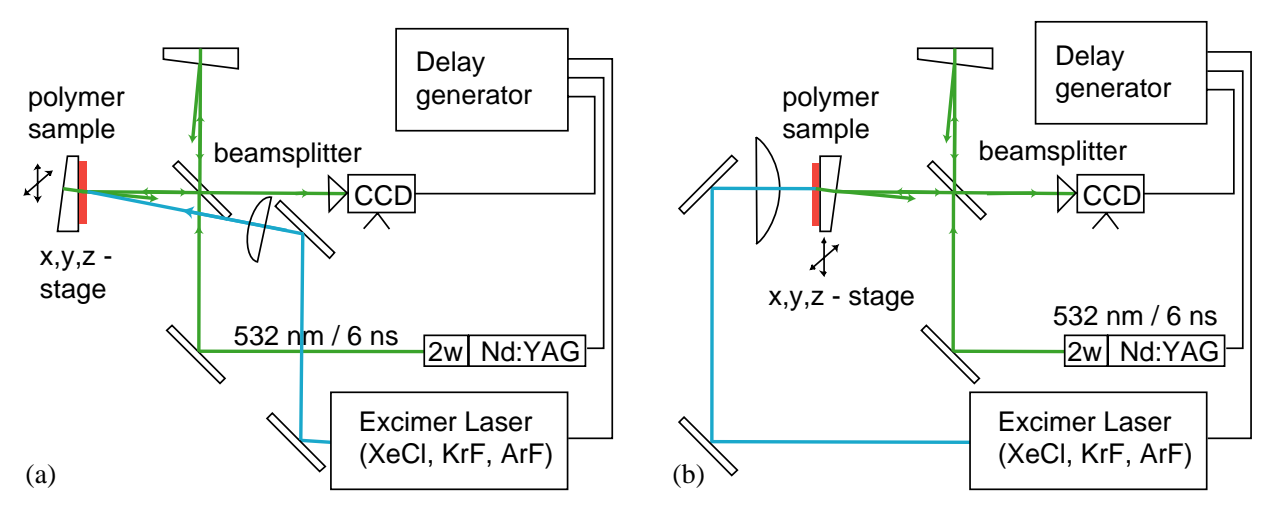

Fig. 1. The two different surface interference setups: (a) front-side interferometer, (b) backside interferometer. 
(b) Backside probe beam: The probe beam passes through the backside of the substrate and is reflected from the polymer/air interface (Fig. 1(b)). In this case, the probe beam is not influenced by the released shockwave and the ejected material. The disadvantage of this variation, is the fact that the substrate and the studied material have to be transparent to the probe beam, and that changes of the refractive index during the irradiation are included in the resulting fringe shifts. The refractive index of the polymer was obtained by comparing the fringe shifts, with the surface profile measured with a surface profilometer (Sloan, Dektak 8000).

For the shadowgraphy experiments also two different setups can be applied:

(a) In the conventional shadowgraphy setup the probe beam $(\mathrm{XeCl}$ or second harmonic of $\mathrm{Nd}$ :YAG) is used to generate fluorescence of a laser dye in a quartz cuvette (see Fig. 2(a)). The cuvette and a camera are mounted perpendicular to the substrate surface. The solid and liquid particles which are released during the ablation process are illuminated by the fluorescence light from the quartz cuvette and are imaged with the camera. The released shockwave causes a local difference of the refractive index, which is visible as a brightened or a darkened ring surrounding the center of the ablation.

(b) The second shadowgraphy setup utilizes a Mach Zehnder interferometric configuration (Fig. 2(b)). In this setup, the probe (second harmonic of Nd:YAG) beam is divided by a beamsplitter $(50 / 50)$. One beam of the interferometer passes parallel to the surface of the polymer through the ablation plume, while the other beam is unaffected by the ablation process. Both beams are recombined in a second beamsplitter and the resulting fringe pattern is imaged by a camera. The refractive indices of the shockwave and the ejected gaseous fragments differ from the noninfluenced atmosphere, resulting in a different optical path length through the plume which causes a phase shift.

The evaluation of the fringe shifts (from surface interference and Mach Zehnder shadowgraphy) was done, according to a procedure described in detail elsewhere

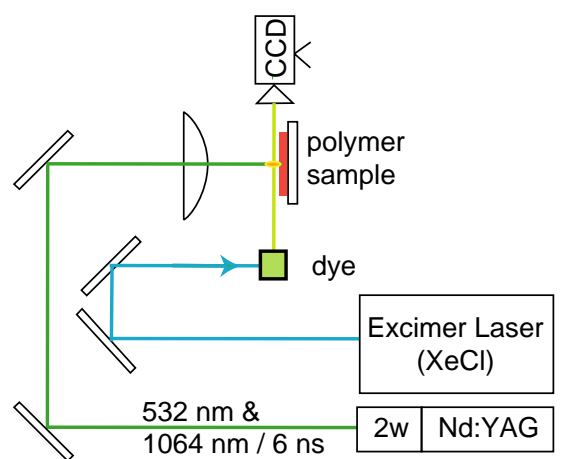

(a)

Fig. 2. The two different shadowgraphy setups: shadowgraphy.

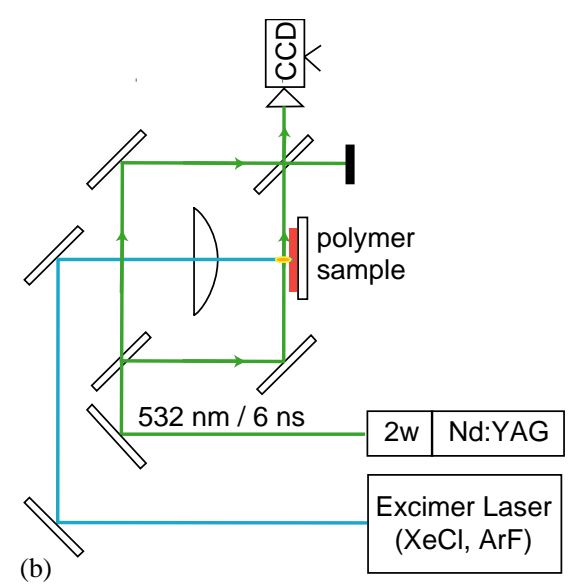

(a) conventional shadowgraphy, (b) interference 

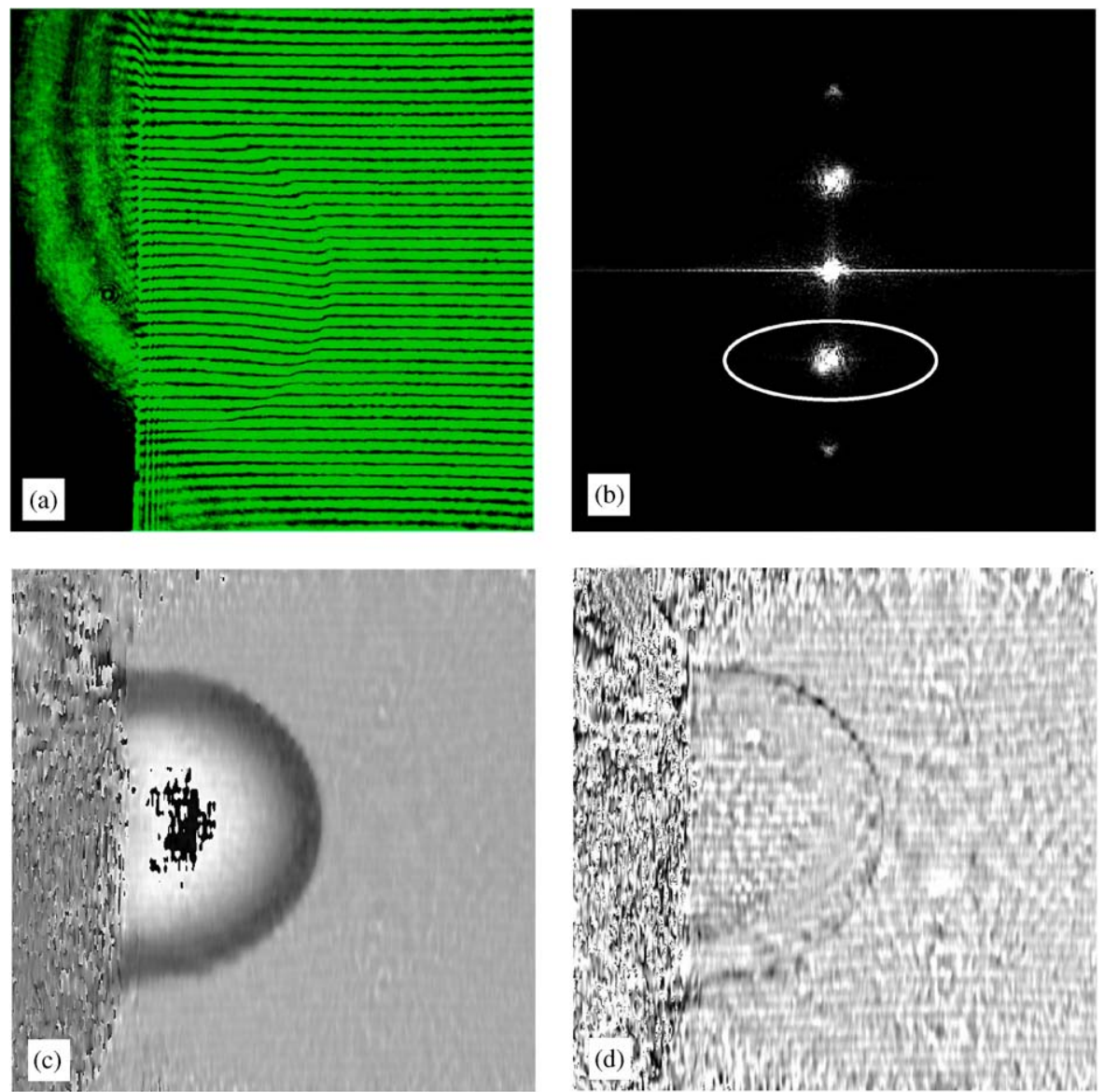

Fig. 3. Data evaluation of the ns-surface interferometry and the interference shadowgraphy: (a) the interference image recorded by the camera; (b) 2D-FFT of the interference image. The ellipse shows the selected peak of the digitally filtering of the image; (c) phase shift image; (d) amplitude image of the released shockwave. The image width is $2.6 \mathrm{~mm}$.

$[27,28]$. Briefly, two images were taken, one before and one during/after the laser pulse (i.e. Fig. 3(a)) and were used for the image analysis. Both images were 2D fast Fourier transformed (2D FFT) (Fig. 3(b)) and reduced to the fringe information by extracting the peak belonging to the fringe shift (white ellipse in Fig. 3(b)). The fringe shift information is then transformed into the complex space using the inverse 2D FFT. The phase difference $(\Delta \varphi)$ of both pictures is then proportional to the changes of the surface morphology $(\Delta d)$ and the refractive index $\left(n_{D}\right)$ (Fig. 3(c)),

$$
\Delta \varphi(\Delta d)=4 \pi \frac{\Delta d n_{\mathrm{D}}}{\lambda}
$$

A phase jump will occur when the difference becomes larger than the dynamic range, i.e. $2 \pi$. It is very important to take these phase jumps during the data 
evaluation into account. In the images obtained from the Mach-Zehnder shadowgraphy, a sudden change of the phase shift, which corresponds to the shockwave front, is observed. The propagation velocity of the shockwave can be determined by measuring the position of the shockwave and applying the time delay.

The amplitude difference between both pictures is proportional to the difference of the amount of reflected light (ns-interferometry), and the amount of transmitted light (ns-shadowgraphy) (Fig. 3(d)), respectively. It is therefore possible to obtain reflectivity and surface morphology changes during the same interferometer experiment.

An increase of the pump laser intensity can result in the formation of a plasma. The processes inside the plasma are, for some applications (i.e. laser plasma thruster), of great interest. The composition of the plasma can be determined by analyzing the light emitted from the plasma plume. This light is collected by a lens, focused into a fiber optic and passed through a monochromator onto a gated intensified charged coupled device (ICCD) (ICCD-1024-MDLG-E/1, Princeton Instruments). The gated intensifier allowed the acquisition of time-resolved spectra, while several pulses were accumulated to achieve a good signal-to-noise ratio.

\section{Results and discussion}

Fig. 4, shows the surface displacement and the changes of the surface reflectivity of a triazene polymer after irradiation with a $\mathrm{XeCl}$ excimer laser. The surface displacement was measured using the backside probe beam configuration. The negative phase shift represents surface removal. The laser intensity and the deposited laser energy are also included in the figure.

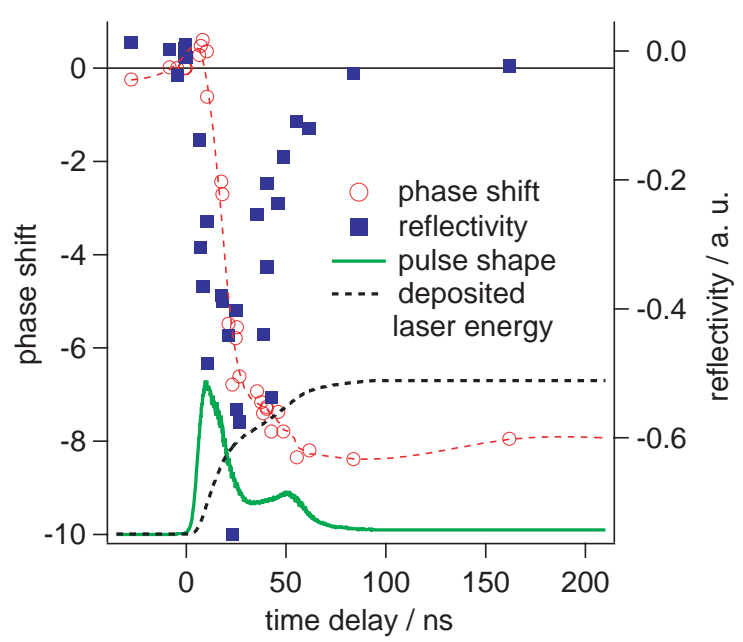

Fig. 4. Reflectivity change during the ablation process of the triazene polymer after irradiation at $308 \mathrm{~nm}$ with $125 \mathrm{~mJ} \mathrm{~cm}^{-2}$. The intensity of the pulse and the displacement of the polymer surface have been included. A smoothed spline was added to guide the eye. 
At the beginning of the laser pulse, a short transient positive phase shift is observed. The decay of this positive phase shift is too fast to be attributed to a simple thermal expansion. This phase shift may be due to a very dense gas layer, which is formed during the initial steps of the polymer decomposition. For this dense gas layer, it is reasonable to assume that the probe beam might not be reflected from the polymer/gas interface, but from the dense gas layer/atmosphere interface. The expansion of this gas layer results in a decrease of its density. At a certain time, the probe beam will then be reflected by the polymer/gas interface. Another possibility is a transient change of the refractive index of the polymer during the irradiation laser pulse, which would affect the probe beam passing through the polymer.

After the initial positive phase shift, a negative phase shift is observed. This phase shift is assigned to material removal. The temporal behavior of the phase shift changes corresponds quite well to the laser pulse, i.e. the beginning and the end of the phase shift changes correspond to the duration of the laser pulse. This and the absence of a thermal expansion of the polymer are strong indications for a photochemical ablation process.

The reflectivity decreases with the laser pulse and reveals the initial values after approximately $50 \mathrm{~ns}$. No permanent changes of the reflectivity are observed. This is in good agreement with a surface analytical study where no changes of the chemical composition of the ablated polymer surfaces was detected [29]. A decrease of the refractive index $\left(n_{D}\right)$ of the polymer or an increase of the refractive index in front of the polymer can both explain the change of the reflectivity. Other possibilities are a temporary surface roughening, or products that form micro bubbles which increase the amount of diffuse reflected light and thereby decrease the specular reflected light. The existing data are not sufficient to exclude any of the above-described possibilities.

Figs. 5 and 6, show a series of shadowgraphy images taken with the conventional and with a Mach-Zehnder shadowgraphy setup for the energetic polymer. An ArF excimer laser (Mach-Zehnder, Fig. 5) and the fundamental of a Nd:YAG (1064 nm) (conventional setup, Fig. 6) were used as pump lasers. The irradiation at $193 \mathrm{~nm}$ is of special interest, due to the absorption of the polymer, while for the absorption at $1064 \mathrm{~nm}$, the carbon dopant absorbs the laser light. This IR wavelength is of interest for laser plasma thrusters, as these devices utilize IR-laser (diodes). The applied energetic polymer has shown a very good thrust-laser energy ratio $[1,2]$ and is therefore an important material for studies using this irradiation wavelength.

The evaluated images of the Mach-Zehnder shadowgraphy setup (Fig. 5) reveal a sudden phase shift caused by a change of the refractive index behind the shockwave. The shockwave itself corresponds to the sudden change of the phase shift images. The ejection of particles is clearly visible in the images obtained with the conventional shadowgraphy setup (Fig. 6). The shockwave is visible as a bright ring surrounding the ejected plume.

Fig. 7 shows the expansion of shockwave after irradiation at 193 and $1064 \mathrm{~nm}$. The shockwave velocity is higher after $193 \mathrm{~nm}$ irradiation although the laser energy was lower $(1.56 \mathrm{~mJ}$ with a spot diameter of $520 \mu \mathrm{m})$ than the energy of the $1064 \mathrm{~nm}$ irradiation $(2.7 \mathrm{~mJ}$ with a spot diameter of $470 \mu \mathrm{m})$. This indicates that the created 

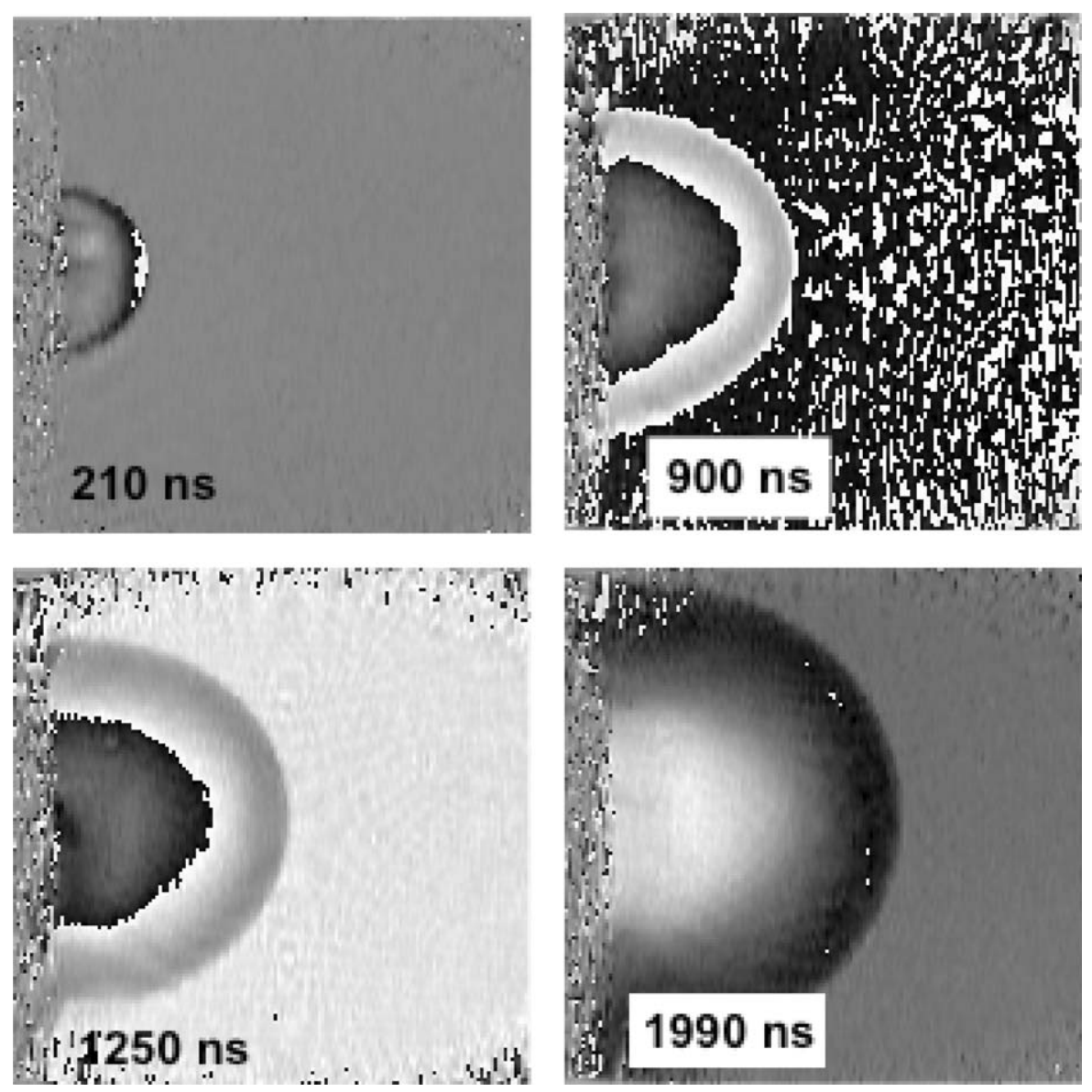

Fig. 5. Interference shadowgraphy phase shift images recorded after irradiation of the energetic polymer with $1.6 \mathrm{~mJ}$ at $193 \mathrm{~nm}$. The image width is $3.8 \mathrm{~mm}$.

pressures and the amount of gaseous products increase with decreasing irradiation wavelength, whereas the amount of solid particles increases (in Figs. 5 and 6) with increasing irradiation wavelength. The density of absorption sites that can be broken increases $\left(\alpha_{\operatorname{lin}, 193 \mathrm{~nm}}=44000 \mathrm{~cm}^{-1}, \alpha_{\operatorname{lin}, 1064 \mathrm{~nm}}=420 \mathrm{~cm}^{-1}\right)$ with the irradiation wavelength. The higher number of broken bonds result in a larger amount of smaller fragments and therefore a faster shockwave velocity.

The penetration depth of the laser light decreases additionally with the decreasing wavelength resulting in higher temperatures in a smaller volume and therefore a more efficient decomposition which results in the creation of more gaseous fragments and the faster shockwave.

This is supported by the predictions of a model describing the planar expansion of shockwaves $[20,30]$. The model suggests that the shockwave velocity should increase with a decrease of the irradiated volume. The shockwave should propagate faster for 

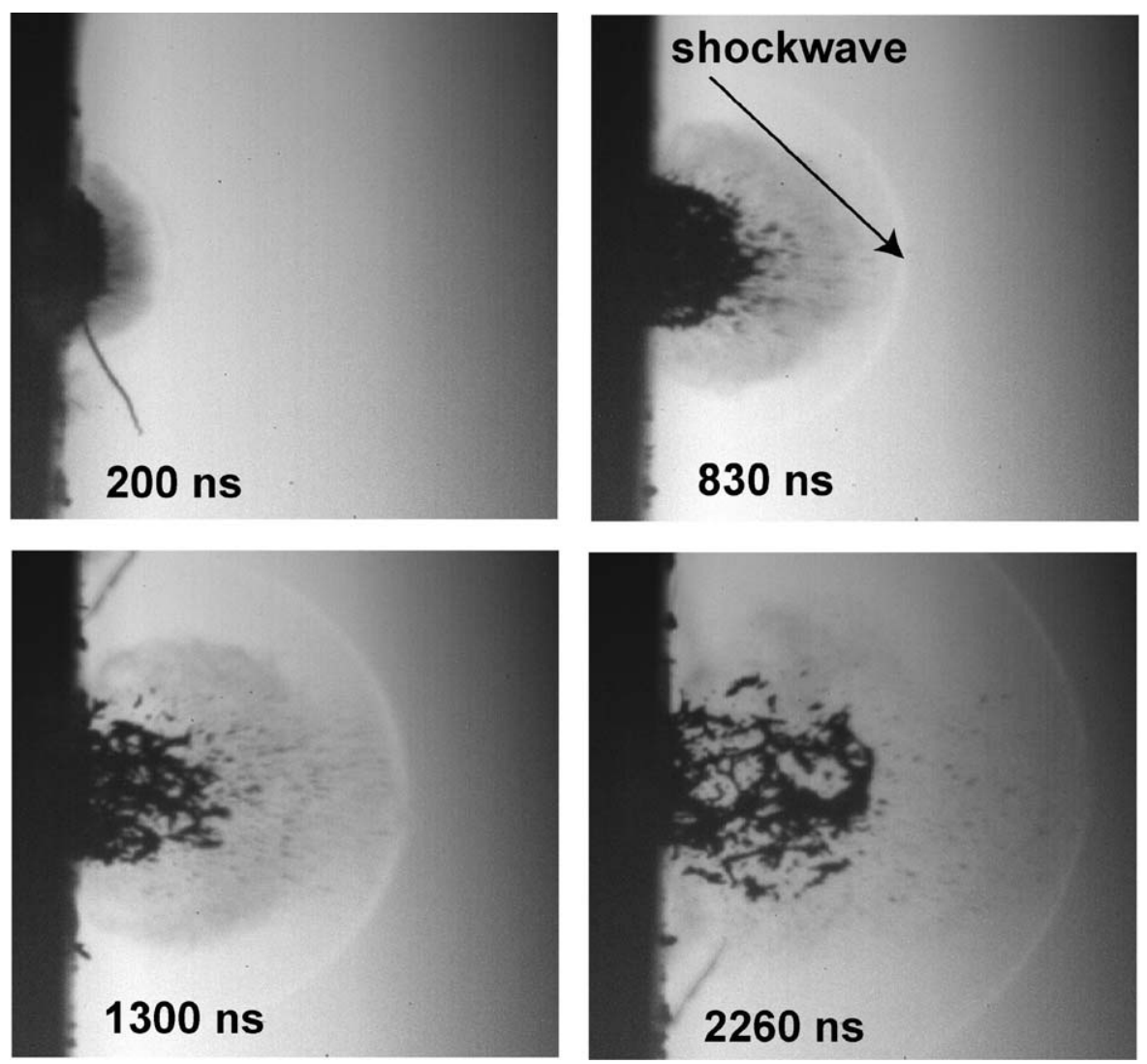

Fig. 6. Images of the shockwave and the ejected material after irradiation of the energetic polymer with $11.2 \mathrm{~mJ}$ at $1064 \mathrm{~nm}$. The image width is $2.7 \mathrm{~mm}$.

the same laser energy and spot size as the penetration depth decreases with the wavelength.

Emission spectroscopy can to be used to study the laser ablation processes at higher fluences, where a plasma is formed. The processes at these fluences are of special interest for laser plasma thrusters. The emission spectra of the energetic polymer, $1 \mu$ s after $1064 \mathrm{~nm}$ irradiation, are shown in Fig. 8. The spectrum reveals the presence of atomic and diatomic species. The complex peak structures could be assigned to diatomic species (CN-Violett around 358, 389 and $416 \mathrm{~nm}, \mathrm{CH}-\mathrm{System}$ around $433, \mathrm{C}_{2}$-Swan around 469 and $514 \mathrm{~nm}$ [21]). The peak around $486 \mathrm{~nm}$ is due to the presence of hydrogen and represents the Balmer- $\beta$ line.

Spectra taken at different delay times (not shown) reveal that at early times $(<1 \mu \mathrm{s})$, a broad emission due to Bremsstrahlung dominates the spectrum. This emission decays within 1-2 $\mu$ s and the specific peaks are clearly visible. The atomic hydrogen peaks disappear within $4 \mu$ s after the laser pulse, while the diatomic peaks can be observed for up to $40-50 \mu$ s. 


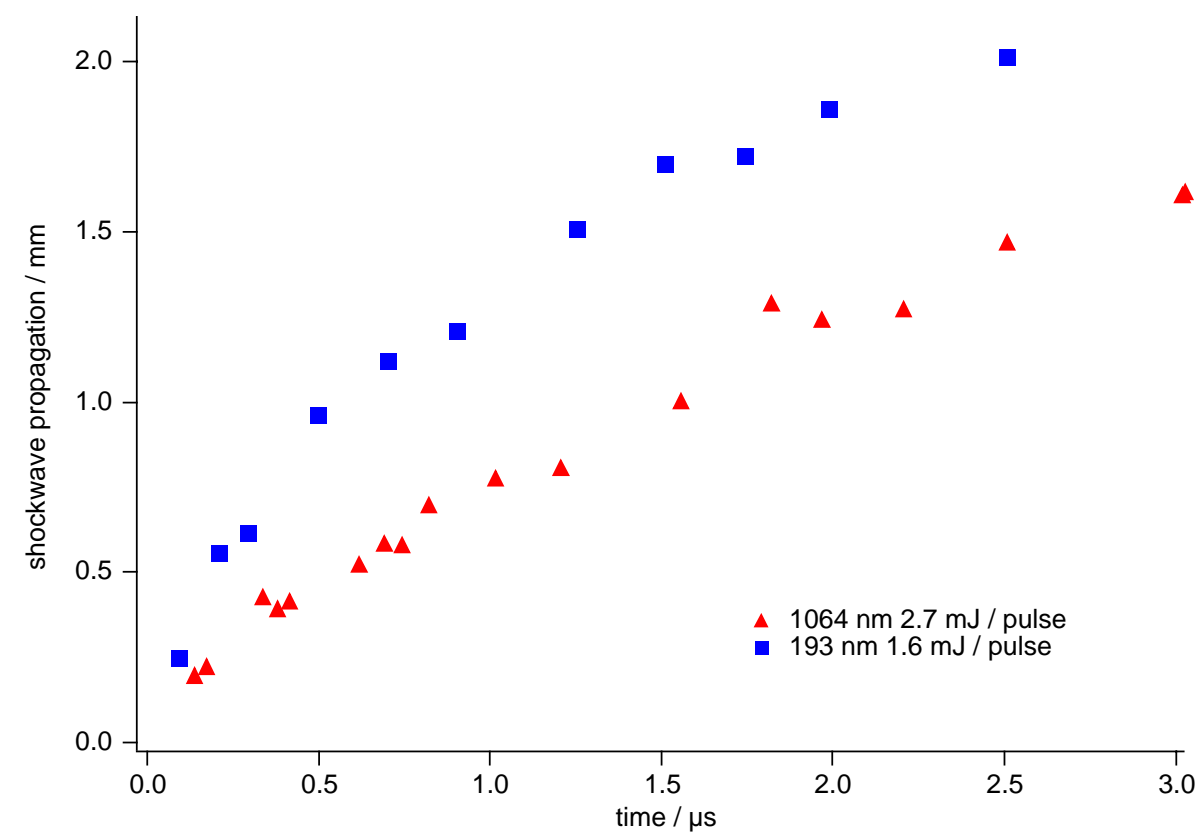

Fig. 7. Expansion of the shockwave of the energetic polymer after irradiation with $1064 \mathrm{~nm}$ and $193 \mathrm{~nm}$.

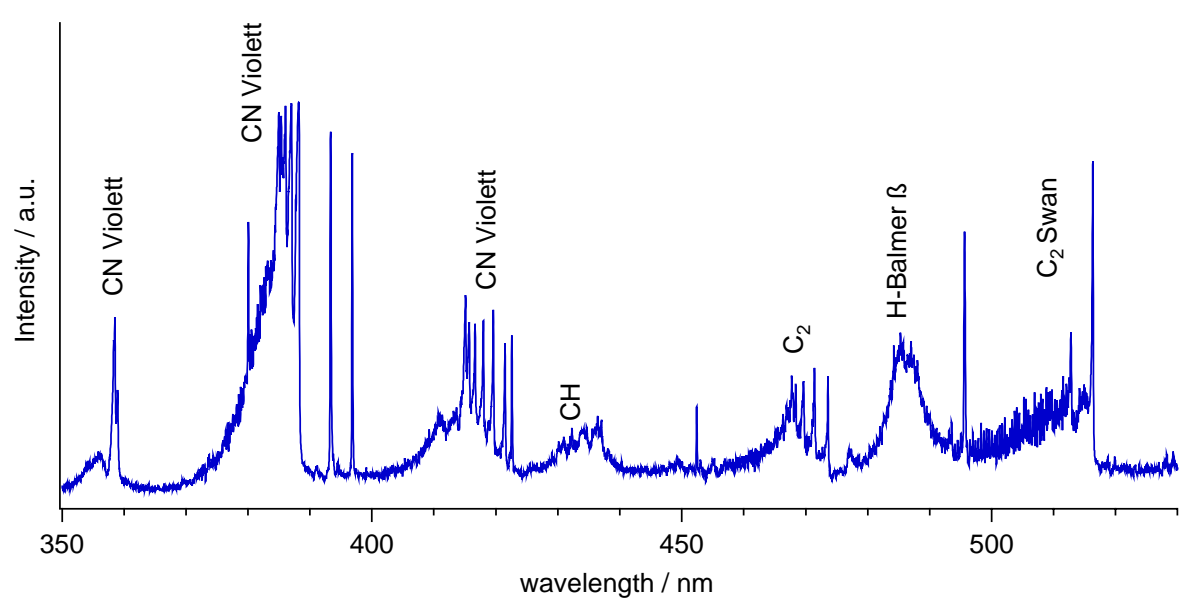

Fig. 8. Emission of the plasma $1 \mu$ s after the irradiation of the energetic polymer with $15.5 \mathrm{~mJ}$ at $1064 \mathrm{~nm}$.

By modeling [31], the fine structure of the CN-Violett system around $489 \mathrm{~nm}$ and comparing it to the measured spectrum, it is possible to determine the ro-vibrational temperature of the $\mathrm{CN}$-species inside the plasma. A temperature of about $7100 \mathrm{~K}$ was calculated for laser energies of $15.5 \mathrm{~mJ}$ and a delay of $1 \mu \mathrm{s}$. 


\section{Conclusion}

Time-resolved ns-interferometry is a useful method to observe the surface morphology changes during and after the laser pulse. The special designed photopolymer reveals no thermal surface expansion, with material removal only observed during the irradiation laser pulse. A transient decrease of the surface reflectivity during the laser pulse was also detected.

Two different setups were applied for ns-shadowgraphy. The conventional setup is especially useful to gain information about the amount of non-gaseous material ejected in the ablation process. The Mach-Zehnder setup requires a more complicated data evaluation, but the shockwaves can be detected with a higher sensitivity. With both methods, the propagation velocity of the shockwave can be determined. Information about the energy contained in the shockwave and the amount of gaseous products released during the ablation process, can be determined from the propagation velocity.

Time-resolved emission spectroscopy allows the detection of various plasma species and the emission from the inverse Bremsstrahlung could be measured. Modelling the fine structure of peaks from diatomic species allows an estimation of the plasma temperature created by the laser ablation process.

\section{Acknowledgements}

Financial support of the Swiss National Science Foundation and EOARD under Contract F61775-01-WE057 are gratefully acknowledged.

\section{References}

[1] Phipps CR, Luke JR, McDuff GG, Lippert T. Laser ablation powered mini-thruster. SPIE 2002;4760:833-42.

[2] Luke JR, Phipps CR, McDuff GG. Laser plasma thruster continuous thrust experiment. SPIE 2002; $4760: 843-51$.

[3] Patel RS, Wassick TA. Laser processes for multichip module's high density multilevel thin film packaging. Proc SPIE-Int Soc Opt Eng 1997;2991:217-23.

[4] Hare DE, Dlott DD. Picosecond coherent Raman-study of solid-state chemical reactions during laser polymer ablation. Appl Phys Lett 1994;64:715-7.

[5] Hare DE, Franken J, Dlott DD. Coherent Raman measurements of polymer thin-film pressure and temperatrue during picosecond laser-ablation. J Appl Phys 1995;77:5950-60.

[6] Lippert T, Stoutland PO. Laser-material interactions probed with picosecond infrared spectroscopy. Appl Surf Sci 1997;109-110:43-7.

[7] Lippert T, Koskelo A, Stoutland PO. Direct observation of a photoinduced Wolff rearrangement in PMMA using ultrafast infrared spectroscopy. J Am Chem Soc 1996;118:1151-2.

[8] Hauer M, Dickinson JT, Langford SC, Lippert T, Wokaun A. Influence of the irradiation wavelength on the ablation process of designed polymers. Appl Surf Sci 2002;197:791-5.

[9] Lippert T, Langford SC, Wokaun A, Savas G, Dickinson JT. Analysis of neutral fragments from ultraviolet laser irradiation of a photolabile triazeno polymer. J Appl Phys 1999;86:7116-22. 
[10] Lippert T, Wokaun A, Langford SC, Dickinson JT. Emission of neutral molecules during UV laser ablation of a photolabile triazeno polymer. Appl Phys A 1999;69:655-8.

[11] Krajnovich DJ. Incubation and photoablation of poly (methyl methacrylate) at $248 \mathrm{~nm}$. New insight into the reaction mechanism using photofragment translational spectroscopy. J Phys Chem A 1997;101:2033-9.

[12] Furutani H, Fukumura H, Masuhara H. Nanosecond time-resolved interferometric study on morphological dynamics of doped poly (methyl methacrylate) film upon laser-ablation. Appl Phys Lett 1994;65:3413-5.

[13] Furutani H, Fukumura H, Masuhara H, Kambara S, Kitagutchi T, Tsukada H, Ozaw T. Laserinduced decomposition and ablation dynamics studied by nanosecond interferometry. 2. A reactive nitrocellulose film. J Phys Chem B 1998;102:3395-401.

[14] Furutani H, Fukumura H, Masuhara H, Lippert T, Yabe A. Laser-induced decomposition and ablation dynamics studied by nanosecond interferometry. 1. A triazenopolymer film. J Phys Chem A 1997;101:5742-7.

[15] Hauer M, Funk DJ, Lippert T, Wokaun A. Laser ablation of a triazene polymer studied by nsinterferometry and shadowgraphy. SPIE-Int Soc Opt Eng 2002;4760:259-68.

[16] Hauer M, Funk DJ, Lippert T, Wokaun A. Laser ablation of polymers studied by ns-interference and ns-shadowgraphy measurements. Appl Surf Sci 2003;208:107-12.

[17] Hauer M, Funk DJ, Lippert T, Wokaun A. Laser induced decomposition of a designed and a commercial polymer studied by ns-interferometry and shadowgraphy. Appl Phys A 2003;77:297-301.

[18] Srinivasan R. Ablation of polyimide (Kapton) films by pulsed (ns) ultraviolet and infrared $(9.17 \mu \mathrm{m})$ lasers-A comparative study. Appl Phys A 1993;56:417-23.

[19] Fukumura H, Takahashi E-I, Masuhara H. Time-resolved spectroscopic and photographic studies on laser-ablation of poly (methyl methacrylate) film doped with biphenyl. J Phys Chem 1995;99:750-7.

[20] Bennett LS, Lippert T, Furutani H, Fukumura H, Masuhara H. Laser induced microexplosions of a photosensitive polymer. Appl Phys A 1996;63:327-32.

[21] Acquaviva S, De Giorgi ML. Temporal and spatial analysis of plasmas during graphite laser ablation in low-pressure $\mathrm{N}_{2}$. Appl Surf Sci 2002;197:21-6.

[22] Wong KH, Tou TY, Low KS. Pulsed $\mathrm{CO}_{2}$ laser ablation of graphite and polymers. J Appl Phys 1998;83:2286-90.

[23] Davis GM, Gower MC, Fotakis C, Efthimiopoulus T, Agyrakis P. Spectroscopic studies of ArF laser photoablation of PMMA. Appl Phys A 1985;36:27-30.

[24] Wei J, Hoogen N, Lippert T, Nuyken O, Wokaun A. Novel laser ablation resists for excimer laser ablation lithography. Influence of photochemical properties on ablation. J Phys Chem B 2001;105:1267-75.

[25] Lippert T, David C, Dickinson JT, Hauer M, Kogelschatz U, Langford SC, Nuyken O, Phipps C, Robert J, Wokaun A. Polymers for UV and near-IR irradiation. J. Photochem. Photobiol A Chem 2001;145:145-57.

[26] Luke JR, Phipps CR, McDuff GG. Laser plasma thruster continuous thrust experiment. Proc SPIEInt Soc Opt Eng 2002;4760:843-51.

[27] Gahagan KT, Moore DS, Funk DJ. Ultrafast interferometric microscopy for laser-driven shock wave characterization. J Appl Phys 2002;92:3679-82.

[28] Takeda M, Ina H, Kobayashi S. Fourier-transform method of fringe-pattern analysis for computerbased topography and interferometry. J Opt Soc 1982;72:156-60.

[29] Lippert T, Nakamura T, Niino H, Yabe A. Laser induced chemical and physical modifications of polymer films: dependence on the irradiation wavelength. Appl Surf Sci 1996;109-10:227-37.

[30] Freiwald DA. Approximate blast wave theory and experimental data for shock trajectories in a linear explosive-driven shock tubes. J Appl Phys 1972;43:2224-6.

[31] Luque J, Crosley DR. LIFBASE: database and spectral simulation program. SRI international report MP 99-009. 1.5 1.5, 1999. 